\title{
The Maia detector array and X-ray fluorescence imaging system: Locating rare precious metal phases in complex samples
}

\author{
C.G. Ryan ${ }^{* a}$, D.P. Siddons ${ }^{\mathrm{b}}$, R. Kirkham ${ }^{\mathrm{c}}$, Z.Y. Li ${ }^{\mathrm{b}}$, M.D. de Jonge ${ }^{\mathrm{d}}$, D. Paterson ${ }^{\mathrm{d}}$, J.S. Cleverley ${ }^{\mathrm{a}}$, A. \\ Kuczewski $^{\mathrm{b}}$, P.A. Dunn ${ }^{\mathrm{c}}$, M. Jensen ${ }^{\mathrm{c}}$, G. De Geronimo ${ }^{\mathrm{b}}$, D.L. Howard ${ }^{\mathrm{d}}$, B. Godel ${ }^{\mathrm{a}}$, K.A. Dyl, ${ }^{\mathrm{a}, \mathrm{f}}$, L.A. \\ Fisher $^{\mathrm{a}}$, R.H. Hough ${ }^{\mathrm{a}}$, S.J. Barnes ${ }^{\mathrm{a}}$, P.A. Bland ${ }^{\mathrm{f}}$, G. Moorhead ${ }^{\mathrm{c}}$, S.A. James ${ }^{\mathrm{d}}$, K.M. Spiers ${ }^{\mathrm{d}}$, G. \\ Falkenberg $^{\mathrm{e}}$, U. Boesenberg ${ }^{\mathrm{e}}$ and G. Wellenreuther ${ }^{\mathrm{e}}$ \\ ${ }^{a}$ CSIRO Earth Sciences and Resource Engineering, 26 Dick Perry Avenue, Kensington, WA 6151, \\ Australia; ${ }^{b}$ Brookhaven National Laboratory, Brookhaven, Upton NY, USA; ${ }^{\mathrm{c} C S I R O}$ Materials \\ Science and Engineering, Normanby Road, Clayton VIC 3168, Australia; ${ }^{\mathrm{d} A u s t r a l i a n}$ Synchrotron, \\ Clayton VIC, Australia; ${ }^{~}$ HASYLAB, DESY, Notkestr. 85, D-22603 Hamburg, Germany; \\ ${ }^{f}$ Department of Applied Geology, Curtin University, GPO Box U1987, Perth, WA 6845, Australia.
}

\begin{abstract}
$\mathrm{X}$-ray fluorescence images acquired using the Maia large solid-angle detector array and integrated real-time processor on the X-ray Fluorescence Microscopy (XFM) beamline at the Australian Synchrotron capture fine detail in complex natural samples with images beyond $100 \mathrm{M}$ pixels. Quantitative methods permit real-time display of deconvoluted element images and for the acquisition of large area XFM images and 3D datasets for fluorescence tomography and chemical state (XANES) imaging. This paper outlines the Maia system and analytical methods and describes the use of the large detector array, with a wide range of X-ray take-off angles, to provide sensitivity to the depth of features, which is used to provide an imaging depth contrast and to determine the depth of rare precious metal particles in complex geological samples.
\end{abstract}

Keywords: Maia detector, X-ray fluorescence microscopy, synchrotron X-ray fluorescence, real-time element imaging, quantitative SXRF analysis, depth mapping, platinum group minerals, Allende meteorite

\section{INTRODUCTION}

The need to capture intricate chemical spatial distribution detail in complex natural samples at high resolution has provided the motivation for the development of the Maia X-ray microprobe imaging system ${ }^{1}$. The aim has been to record element concentration variations limited only by the X-ray micro/nano-probe beam size over the spatial scales demanded by the science and in practical timeframes. In earth science applications, sample sizes for detailed microscopy and mineralogy are typically $\sim 40-70 \times 25 \mathrm{~mm}^{2}$ thin-sections selected to capture chemical features and textural detail that provide clues to mineralization and geochemical processes. Target features may occur as very rare sub-micron minerals or precious metal particles, such as gold or platinum group minerals (PGMs). These "needle in a haystack" scenarios are a particular challenge.

Focused synchrotron $\mathrm{X}$-rays or $\mathrm{MeV}$ energy ion probes are well suited to detect these minute objects, as their penetration enables the particles to be excited and imaged at depth below the surface of the section. This greatly enhances their probability of detection over (near-) surface constrained probes. The Kirkpatrick Baez (KB) mirror end-station of the Xray Fluorescence Microscopy (XFM) beamline at the Australian Synchrotron ${ }^{2}$ provides a monochromatic $2 \mu \mathrm{m}$ beamspot size for energies in the range 4-20 keV. Equipped with the Maia system, the XFM beamline can acquire images at $2 \mu \mathrm{m}$ resolution over areas of several square centimeters ${ }^{\dagger}$. This leads to image sizes that can exceed $10^{8}$ pixels. For image acquisition in a reasonable time (e.g. 10 hours or less) this means pixel dwell times as low as $\sim 0.1-0.3 \mathrm{~ms}$. To achieve

* chris.ryan@csiro.au; phone +61 39545 2090; nmp.csiro.au

${ }^{\dagger}$ The XFM nanoprobe endstation provides 0.1-0.3 $\mu \mathrm{m}$ beam-spots for finer detail over sub-mm scale features.

X-Ray Nanoimaging: Instruments and Methods, edited by Barry Lai, Proc. of SPIE Vol. 8851, 88510 Q (C) 2013 SPIE · CCC code: 0277-786X/13/\$18 · doi: 10.1117/12.2027195 
reasonable counting statistics in a pixel spectrum, at least for the major elements or scattering, demands total count rates of $\sim 4-10 \mathrm{M} / \mathrm{s}$ or more.

To meet this challenge, the philosophy of Maia design has been to (i) spread this count rate over a large number of small detectors, (ii) maximize collection solid-angle to achieve the desired data-rates while minimizing X-ray dose and potential radiation damage to sensitive samples, (iii) closely integrate sample stage encoders and beam flux accumulators with detector data acquisition, and (iv) eliminate readout delay overheads using an event-by-event real-time processing approach. The latter also provides an opportunity for real-time spectral deconvolution and display of element images.

\section{MAIA DETECTOR DESCRIPTION}

Large solid angle is achieved using an upstream annular detector array, with the beam passing perpendicularly through the array and onto the sample. This normal incidence geometry keeps the sample motion plane clear and can accommodate samples and sample arrays of any size. Normal incidence does increases the capture of scattered X-rays compared to a detector located at $90^{\circ}$ to a horizontally polarized synchrotron beam. However, in many applications the image formed from this scattered signal is useful in its own right.

The array comprises 384 planar $1 \times 1 \mathrm{~mm}^{2}$ detectors in an annular configuration $(20 \times 20$ with 16 omitted in the centre; see Figure 1) fabricated by BNL in $0.4 \mathrm{~mm}$ thick high-resistivity silicon ${ }^{3}$, mounted on an aluminum nitride frame and Peltier cooled to $-25^{\circ} \mathrm{C}$. The beam passes through the array via a tapered molybdenum collimator which extends to a beryllium window on the sample side of the detector. The sample plane is located $10 \mathrm{~mm}$ from the array, which thus subtends a solid-angle of $1.3 \mathrm{sr}$.

A molybdenum mask, glued to the front face of the detector wafer, shields the regions between detector pads from fluorescence photons and thus prevents charge-sharing between detectors resulting from lateral diffusion of carriers. This maintains high peak to background ratios in spectra at the expense of some loss of efficiency. The mask comprises three layers, each $100 \mu \mathrm{m}$ thick, with offset rectangular collimation apertures arranged to collimate X-rays originating from the source position $10 \mathrm{~mm}$ above the detector plane ${ }^{4}$.

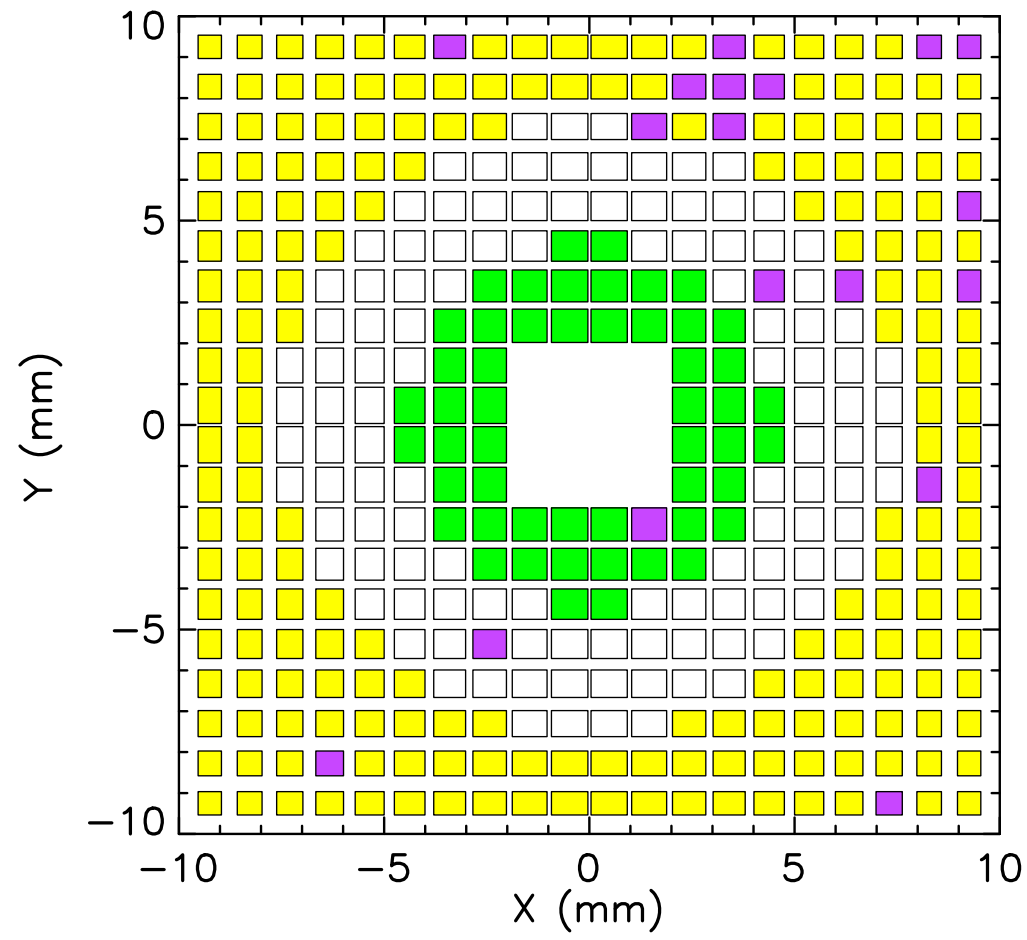

Figure 1 Layout of the Maia 384 revision A detector array at the XFM beamline showing the masked effective size of detectors. Shaded detectors show 51 "inner" (green), 199 "outer" (yellow) (see text) and 18 disabled channels (violet). 
Pads on the detector wafer are directly wire-bonded to twelve CMOS application specific integrated circuits (ASICs) called HERMES, each providing 32 low-noise preamplifiers with high order quasi-Gaussian shapers and baseline stabilizers $^{3}$. Shaped pulses go to twelve 32-channel peak-detector derandomizer ASICs called SCEPTER, which capture pulse height, representing energy (E), and time-over-threshold (T), which is later used for dead-time measurement and pile-up detection and rejection ${ }^{5}$. E and $\mathrm{T}$ analogue pulse outputs from SCEPTER are digitized by dual 14-bit fast synchronous analogue to digital convertors (ADC), with 3 SCEPTERs multiplexed into each ADC pair. A fieldprogrammable gate-array (FPGA) in the detector head transmits photon events $(\{\mathrm{E}, \mathrm{T}, \mathrm{n}\}$, for detector ' $\mathrm{n}$ ') over a dedicated $8 \mathrm{~Gb} / \mathrm{s}$ quad-channel fibre optic link to the processing subsystem located outside the radiation enclosure ${ }^{1}$.

The processing subsystem is based on the parallel processing engine HYMOD, developed at CSIRO for high speed instrumentation. HYMOD provides a Xilinx Virtex 5 FPGA connected to 6 large static RAMs, a PowerPC 8555 processor and fast serial $(12 \times 3.125 \mathrm{~Gb} / \mathrm{s})$ and Ethernet ports $(2 \times 1 \mathrm{~Gb} / \mathrm{s})$. FPGA code is written using a CSIROdeveloped pipelined, parallel high-level processing language called 3PL, which generates a FPGA netlist for Xilinx place-and-route tools. The PowerPC handles logging data and system control and configuration through a TCP/IP network. Direct digital connections to the HYMOD FPGA include stage incremental encoder and pulse counting inputs.

Events resulting from photon interactions at the detector are processed by the HYMOD processing pipeline on each clock cycle, typically at $50 \mathrm{MHz}$. The pipeline comprises: (i) \pm 0.5 dither applied to $\mathrm{E}$ to reduce subsequent aliasing effects, (ii) linearization correction, (iii) linear gain trimming to match $\mathrm{E}$ and $\mathrm{T}$ across detector channels, (iv) energy calibration mapping, (v) pile-up rejection, (vi) individual detector count-rate monitoring, (vii) dead-time and pileup monitoring across the array, and (viii) real-time element image accumulation triggered directly by sample stage motion. Processed events, optionally sub-sampled ('throttled') on the basis of energy, are also logged to disk, tagged by sample stage pixel coordinates and incident and transmitted flux counts accumulated in that pixel. Throttling reduces the size of raw data files through suppression of strong element lines or scatter peaks. Further hardware details are provided by Kirkham et al. ${ }^{1}$.

Recent enhancements to the Maia system, embodied in revision B of the core detector module, include improved energy resolution (Mn Ka FWHM: $255 \mathrm{eV}$ mode at $2 \mathrm{M} / \mathrm{s}$ count-rate, $80 \%$ of detectors less than $275 \mathrm{eV}$ ), achieved through close attention to circuit board layout and grounding. Further energy resolution improvements are being addressed though new ASIC designs.

\section{QUANTITATIVE ANALYSIS AND IMAGING}

The projection of element images using Maia builds on a fundamental parameters approach to quantification. Central to this approach is modelling the generation of X-rays within the sample, specified as a layered structure, the absorption of these X-rays in the sample and external filters on the way to the detector array, collimation by the detector mask, and absorption in the detector. It is implemented in the GeoPIXE code using the approach of Reuter et al. ${ }^{6}$, including secondary fluorescence contributions, and using the sub-shell absorption cross-sections of Ebel et al. ${ }^{7}$, and the CosterKronig transition rates for the $\mathrm{L}$ shell, fluorescence yields and branching ratios from the fundamental parameter database of Elam et al. ${ }^{8}$ with refinements recommended by Campbell ${ }^{9}$. Each detector has a unique effective take-off angle from the sample and solid-angle dictated by mask collimators, angle and distance from the sample and intrinsic efficiency tied to the angle of incidence of X-rays into the detector. This results in a wide range of relative sensitivities for detectors across the array ${ }^{4}$. In GeoPIXE, modelling of yields is done for all lines for each element so that the inferred relative line intensities reflect integration through the sample and variation across the array.

For the analysis of an X-ray fluorescence spectrum, the fundamental parameters modelling of X-ray yields is combined with a non-linear least squares fitting approach that uses the model X-ray line intensities ${ }^{10,11}$. Construction of element images uses the Dynamic Analysis (DA) method ${ }^{12,4}$, which is based on the expression of a linear least-squares fit to an $\mathrm{X}$-ray spectrum as the solution of a set of simultaneous equations, written in matrix form, and re-arranged into the form of a matrix transformation of the spectrum to element concentration vector ${ }^{13,14}$. The linear fit follows from the non-linear fit to a representative spectrum after non-linear parameters representing energy calibration and peak width variation have been determined and fixed.

The DA matrix transformation can be applied to each event in turn, which lends itself to event-by-event processing and a real-time implementation ${ }^{15}$. In off-line analysis using parallel processing in GeoPIXE, event-by-event processing enables processing 3-4 orders of magnitude faster than pixel spectrum fitting. In Maia, DA is implemented in the HYMOD FPGA code as an energy calibration linear mapping and a DA matrix look-up table operation to accumulate 
contributions to element images for each event in real-time. These are output on each pixel advance for real-time display. Full composition correction to account for differences in sample composition per pixel between that assumed for yield modeling and that found in the images can be performed as a post imaging correction. It is an iterative procedure that converges quickly ${ }^{14}$.

Characterization of Maia using standard metal foil targets show that this modelling, together with real-time solutions to flux accumulation, pileup rejection and dead-time correction, provide consistent analyses using individual detectors with a relative standard deviation less than $3 \%$ for total array count rates of at least $12 \mathrm{M} / \mathrm{s}^{16}$.

\section{DEPTH SENSITIVITY}

The large size of the detector array, located just $10 \mathrm{~mm}$ from the beam spot on the sample, provides a large range of takeoff angles at the sample surface, with the angle between sample normal and ray to masked detector centre varying between $13.9^{\circ}$ and $52.6^{\circ}$. This makes the outer detectors much more sensitive to self-absorption for X-rays from a particle at depth in a sample than the inner detectors. Such a difference can be harnessed to provide an imaging contrast based on particle depth and also a quantitative measure of depth for discrete identified particles.

The variation in model yields across the array is illustrated in Figure 2, which shows the yield (counts/ppm/ $\mu \mathrm{C}$ ) ${ }^{\ddagger}$ of $\mathrm{Pt} \mathrm{L} \alpha$ $\mathrm{X}$-rays from a buried particle for individual detectors plotted as a function of the radial distance of each detector from the centre of the array (beam axis). Each symbol type represents the yield from a $1 \mu \mathrm{m}$ layer of Pt at various depths within an olivine sample composition.

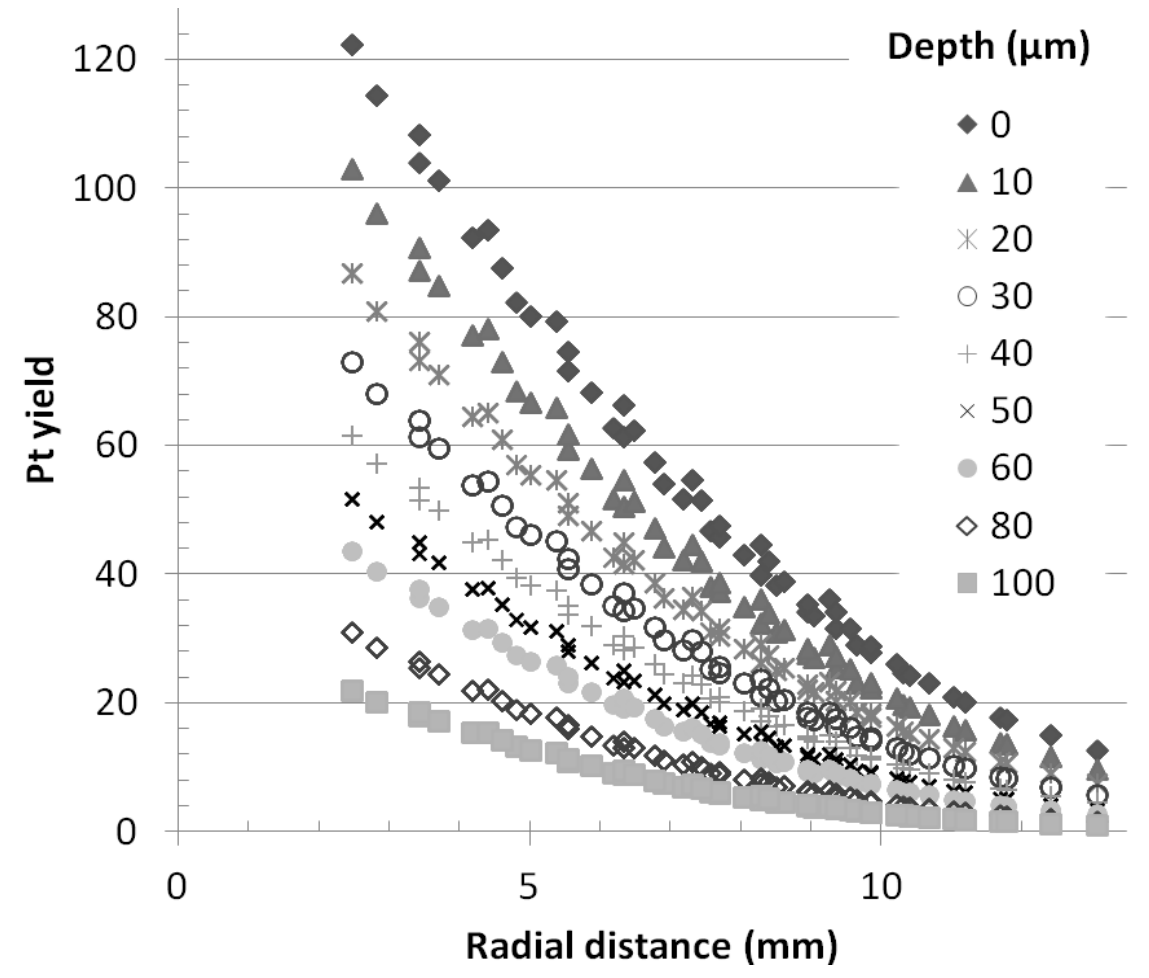

Figure 2 Yield (counts/ppm/ $\mu \mathrm{C}$ ) of Pt L $\alpha$ X-rays from a small Pt particle (modeled as a $1 \mu \mathrm{m}$ buried Pt layer in olivine) versus radial position of individual Maia detectors for various depths $(\mu \mathrm{m})$ to the particle. Note that many points represent multiple detectors at symmetry equivalent positions around array.

Subtle absorption-related effects of particle shape versus a simple layer have been ignored in this discussion. The interesting apparent scatter in these points reflects the non-equivalent positions and orientations of detectors around the

$\star \mu \mathrm{C}$ is a convenient unit used to represent $6.2415 \times 10^{12}$ photons. 
square array. Masking, distance and angle gradually reduces the efficiency of the more peripheral detectors. Depth to the Pt layer has a marked effect on yields, with stronger attenuation seen in the more peripheral detectors.

Combining yields for 51 "inner" and 199 "outer" detectors as shown in Figure 1, with the total numbers chosen to give a similar total yield for a surface feature, produces the curves in Figure 3. The ratio of "outer"/“inner" yields produces a good contrast with the depth of the particle. This can be applied to estimate the depth of small particle features seen in $\mathrm{XY}$ maps, given the assumption that they are similarly localized in the depth dimension.

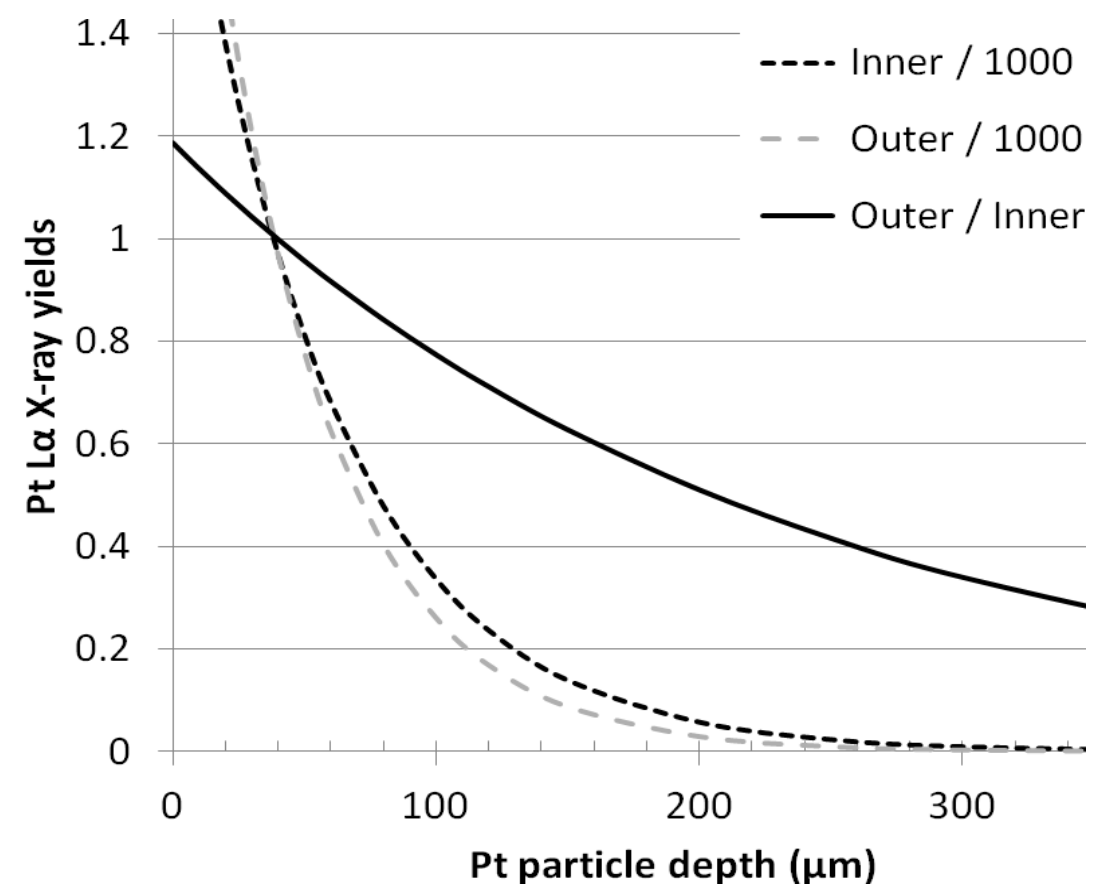

Figure 3 Yields of Pt L $\alpha$ X-rays (counts $/ \mathrm{ppm} / \mu \mathrm{C}$ ) summed over "inner" and "outer" rings of detectors (see Figure 1) scaled by 0.001 , as a function of Pt particle depth $(\mu \mathrm{m})$ in olivine, and the ratio of "outer" to "inner" yields.

\section{APPLICATION METHODS}

The key capabilities of the Maia detector system that enable the high throughput of the XFM beamline are (i) transit times per pixel (referred to below as "dwell time") down to $50 \mu \mathrm{s}^{12}$, (ii) close detection geometry providing a solid angle of $1.3 \mathrm{sr}^{4}$, (iii) high sustained count rates, normally up to $12 \mathrm{M} / \mathrm{s}$, (iv) $100 \times 100 \mathrm{~mm}^{2}$ scan range and image area, (v) image pixel count beyond $10^{8}$ pixels, (vi) real-time spectral deconvolution and image display, and (vii) moderate inelastic scattering signal that provides good collective images of light elements ${ }^{17}$, especially useful for biological samples ${ }^{18-21}$. Typically, pixel times and image areas are not generally a limitation, and these are chosen to suit a particular application. This has enabled a range of application at the XFM beamline:

\subsection{Large area mapping}

Scans of complex natural samples can be done over $100-200 \mathrm{~mm}^{2}$ at full spatial resolution of $2 \mu \mathrm{m}$ on the KB endstation. Larger areas to $10 \mathrm{~cm}^{2}$ are typically done at 4-6 $\mu \mathrm{m}$ resolution. Typical image size is $10-100 \mathrm{M}$ pixels acquired over 210 hours $^{12}$; largest image to date is $620 \mathrm{M}$ pixels. Using a collimated beam, larger objects, such as works of art, have been imaged at $50-100 \mu \mathrm{m}$ resolution ${ }^{17}$ over areas up to $600 \times 300 \mathrm{~mm}^{2}$. This method has been applied to studies in the earth $^{22-27}$, environmental ${ }^{28,29}$, medical ${ }^{18,30}$, biological ${ }^{19-21,31-35}$ and material ${ }^{36}$ sciences as well as cultural heritage ${ }^{17}$.

\subsection{Survey and detail mapping}

Large area mapping can be performed at small transit time per pixel to provide overview survey images (e.g. 6M pixels in 1 hour) in major and minor elements from which smaller detail areas are selected for scanning at increased transit time and/or spatial resolution ${ }^{23,29}$. Real-time imaging enables these selections to be made during survey image acquisition. 


\subsection{D fluorescence tomography}

Large pixel count can be recast as a large voxel count for 3D fluorescence tomography, particularly where matrix absorption is low. 5- $\mu \mathrm{m}$ tomographic data have been acquired using a KB mirror pair with $\sim 2 \mu \mathrm{m}$ resolution over $\sim 1$ $\mathrm{mm}$ specimens $^{33}$. Single-slice data can be obtained from highly radiation-sensitive specimens in a matter of minutes ${ }^{19,20,34,35}$. Measurement of the Ge distribution in an intact rice grain with $2 \mu \mathrm{m}$ voxels over $2-3 \mathrm{~mm}$ dimensions utilized high-definition 2D fluorescence tomography, with a measurement time of the order of 3 hours ${ }^{21}$. Maia has enabled fluorescence tomography to become routine; however the ability to measure ever larger specimens now demands a general solution to the self absorption problem (see ref: 37 and references therein).

\subsection{XANES image stacks}

Spatial images can be repeated for a series of beam energies across a selected element absorption edge to provide 100200 energy XANES spectra at each pixel of an image approaching 1M pixels, from which chemical state images can be projected $^{24}$.

\subsection{XANES tomography}

Repeated measurement of single slice tomography at energies across an absorption edge can be used to provide crosssectional chemical state images ${ }^{38}$.

\section{APPLICATION EXAMPLES}

Two examples follow to illustrate "large area mapping" and the "survey and detail" approach. The first is drawn from a study of carbonaceous chondrite meteorites representing the reduced (Vigarano) and oxidized (Allende) CV3 chondrites by Dyl et al. ${ }^{39}$. The primitive CV3 chondrites represent accreted assemblages of the earliest proto-solar system condensates containing calcium-aluminum-rich inclusions (CAIs) and melt droplets or chondrules embedded in a fine ground mass. This accreted assemblage was then modified - to varying degrees in different meteorites - by hydrothermal processing within the parent body, early in Solar System history. The study aims to use the spatial distribution of trace elements and their mineralogical associations to help distinguish between primary accretion of CAIs and chondrules and subsequent planetesimal processes, such as fluid-rock reactions and oxidation ${ }^{39}$.

The complexity of chondrites is clear from the large area mapping of the whole Allende meteorite section in Figure 4a, which was acquired in 4 hours at $2 \mu \mathrm{m}$ spatial resolution over $30 \mathrm{M}$ pixels. The images exhibit a wonderful variety of chondrules each with a distinctive chemistry, alteration and thermal history recorded in their zoned structures and melt globule textures. Figure $4 \mathrm{~b}$ is an enlargement of one of these chondrules (a $3.2 \mathrm{M}$ pixel sub-set of the larger data-set), which illustrates the quality of fine detail obtained on each chondrule in the large area scan. Refractory elements Pt, Os and Ir are seen in many chondrules as small hot-spots despite their low concentration in the bulk ( $\sim 1 \mathrm{ppm})$. Extracted spectra (not shown) have been used to confirm the element identity of hot-spots as seen in the DA images. Figure $4 \mathrm{~d}$ focuses on the boundary between the chondrule and its igneous rim, showing a number of small Pt phases decorating the edge of a Fe-Ni phase, consistent with the affinity of $\mathrm{Pt}$ for solid solution in Fe-alloy during condensation. Figure 4c displays another region at this boundary, which shows $\mathrm{Cu}$ distribution and a rare Ir phase.

The search for rare platinum group element (PGE) alloys in mantle-derived rocks provides a second example that illustrates the survey and detail mapping approach ${ }^{40}$. This study aims to identify rare Os-Ir nugget survivors of early deep-Earth processes ${ }^{41}$ and Pt alloys that may provide evidence for direct precipitation of precious metal grains from magmas, potentially a critical clue to the genesis of valuable Pt ore deposits ${ }^{42-44}$. The use of synchrotron radiation allows probing of a $3 \mathrm{D}$ volume of rock compared to a $2 \mathrm{D}$ section using conventional scanning electron microscopy, increasing by $\sim 100$ times the chance of finding rare-PGE-rich phases. The Maia detector implemented at XFM can expand this search over several $\mathrm{cm}^{2}$ at high resolution. Previous studies have demonstrated detection of $\sim 0.3 \mu \mathrm{m}$ gold particles in a $\sim 1 \mathrm{~cm}^{2}$ scan area ${ }^{12}$.

Figure 5a shows a $1 \mathrm{~cm}^{2}$ survey scan completed in 3.4 hours, which revealed five candidate Pt alloy particles in three groups seen as hot-spots in the Pt map (identified by circles). One of these (\#1) is seen in the enlarged image from the survey data (Figure 5b). Verification detail scans were performed over small areas surrounding these hot-spots with a longer dwell per pixel $(15.6 \mathrm{~ms})$, which provided improved statistics in the extracted spectra and revealed another two weak Pt particle signals. The total acquisition time for the survey scan plus the three detail maps (two shown in Figure 5) was less than 4 hours, which permitted many survey and detail scans sets to be completed in the available beam-time. 

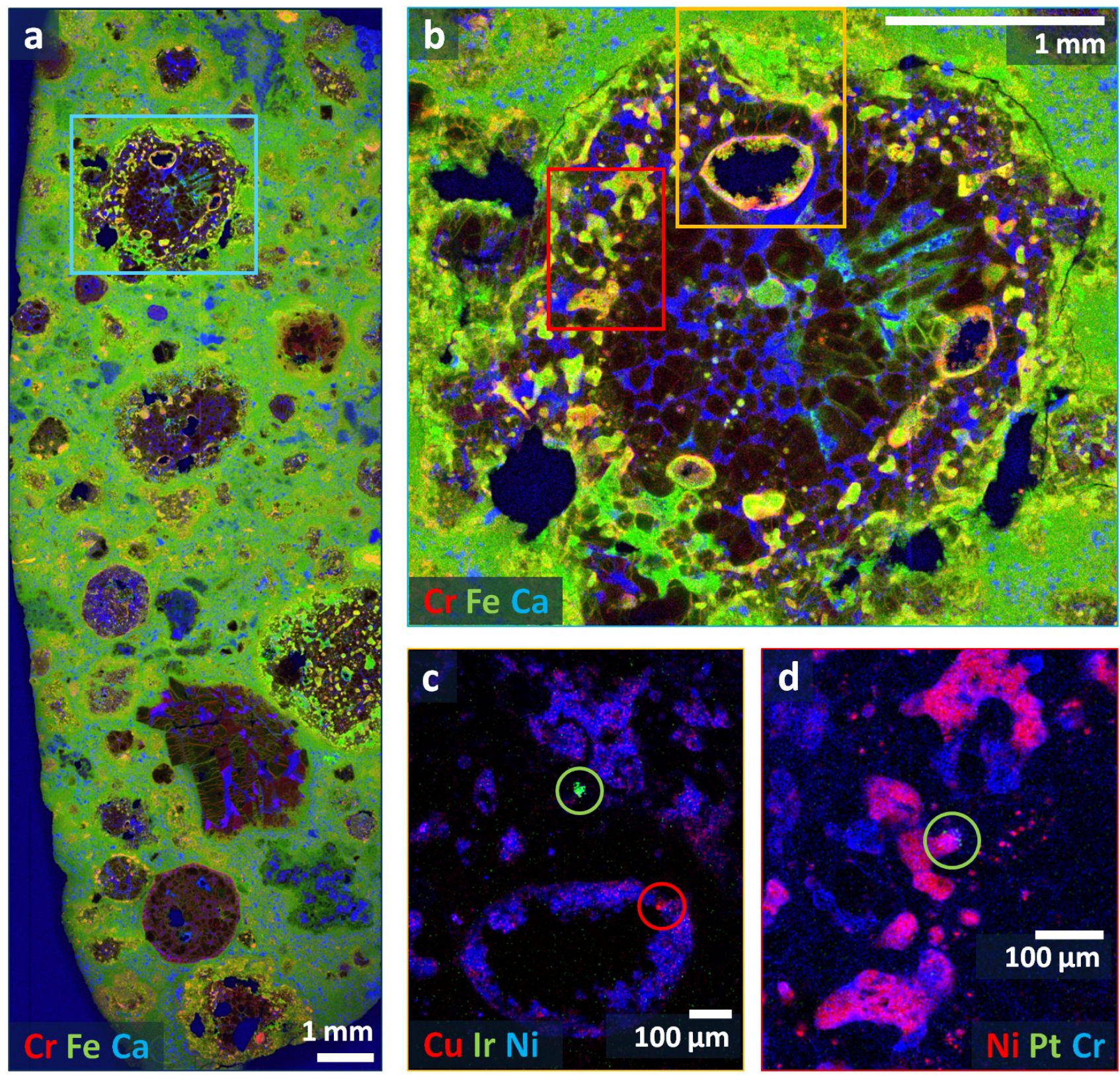

Figure 4 (a) Large area mapping using Maia and the XFM beamline of a polished thin section of Allende 8 carbonaceous chondrite meteorite revealing complex major and trace element structure ${ }^{39}\left(18.5 \times 6.5 \mathrm{~mm}^{2}, 9251 \times 3251\right.$ pixels, $0.49 \mathrm{~ms}$ dwell, 4 hours acquisition at $18.5 \mathrm{keV}$; showing $\mathrm{Cr}$, Fe, Ca as an RGB image); (b) enlargement of a selected chondrule (blue box in (a)); (c) enlargement of chondrule (orange box in (b)) showing $\mathrm{Cu}, \mathrm{Ir}$, Ni as an RGB image; (d) enlargement of chondrule (red box in (b)) showing Ni, Pt, Cr as an RGB image (note decoration of Pt-rich phases in green circle). $\mathrm{Cu}$ and Ir have been averaged over a 4-pixel neighborhood. All images have been rotated $90^{\circ}$ post acquisition. 

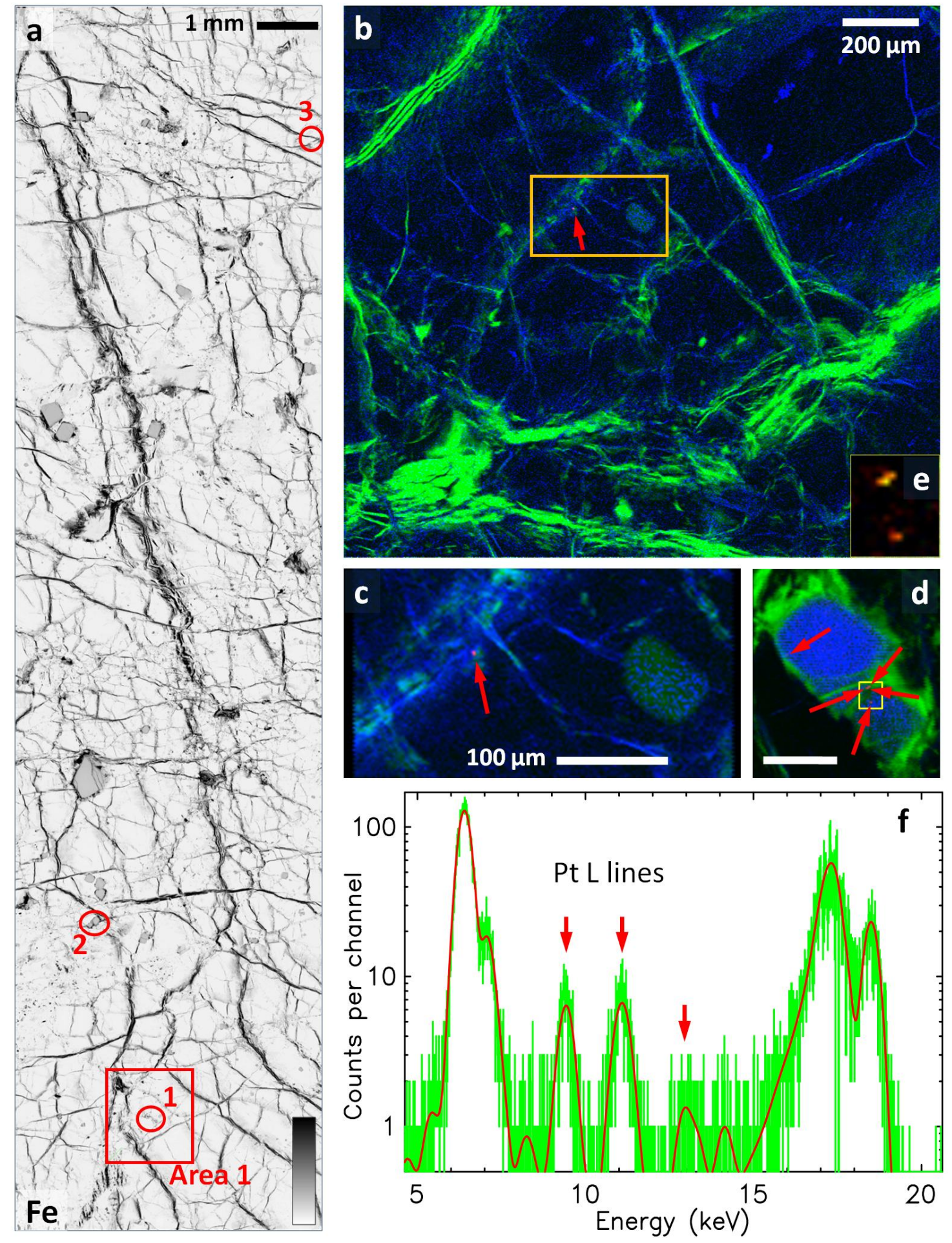

Figure 5 (a) Survey scan using Maia (Fe image) of a polished rock section from Muang Pha intrusion Laos $\left(20 \times 5 \mathrm{~mm}^{2}\right.$, $10002 \times 2502$ pixels, $0.49 \mathrm{~ms}$ dwell, 3.4 hours acquisition at $18.5 \mathrm{keV}$ ) showing Fe map (rotated $90^{\circ}$ ) and identified Pt hotspots in circles; (b) enlargement of "Area 1" in RGB (Pt, Fe, Mn) showing detail scan for Pt \#1 (orange box); (c) detail scan of Pt \#1 (orange box in (b); $360 \times 190 \mu \mathrm{m} ; 15.6 \mathrm{~ms}$ dwell) in RGB (Pt, Fe, Mn); (d) detail scan of Pt \#2 $(250 \times 280 \mu \mathrm{m} ; 15.6$ ms dwell in RGB (Pt, Fe, Mn), scale bar $100 \mu \mathrm{m}$ ) with Pt hot-spots indicated by arrows; (e) enlargement from (d; yellow box) displayed as a depth map in RGB with red proportional to "inner" signal, green proportional to "outer" and zero blue; and (f) sum spectrum from 51 "inner" detectors for Pt \#1, integrated over two pixels, showing clear Pt L line groups. 
Using a threshold on the Pt maps localizes the signal to two pixels in grain Pt \#1 (Figure 5c). Comparison between the Pt signal extracted from the "inner" and "outer" detector groups (Figure 1) produces a ratio of $1.072 \pm 0.03$. Using the "outer/inner" curve in Figure 3, this ratio maps onto a depth of $23 \pm 6 \mu \mathrm{m}$. Five Pt hot-spots are indicated in region $\mathrm{Pt} \# 2$ (Figure 5d). Figure 5e displays an RGB image with red proportional to the "inner" Pt signal and green proportional to the "outer" signal, with zero blue, to provide a depth color contrast. However, these are now located within chromite mineral phases, which has much stronger absorption for Pt L X-rays than olivine. This absorption scales the depth scale on the "outer/inner" model curve by 0.23 , which produces the depths $44 \pm 8,33 \pm 11,45 \pm 10,66 \pm 10$ and $8 \pm 11 \mu \mathrm{m}$ for the five Pt particles. Knowledge of the depths of the Pt alloy particles will enable polishing or focused ion-beam sputter erosion to bring these to the surface for closer examination using SEM techniques and isotopic analysis.

\section{DISCUSSION AND CONCLUSIONS}

The real-time event-by-event processing approach used in the Maia system relaxes constraints on the selection of dwell time per pixel. Image sizes and pixel dwell can be chosen to suit the needs of the application and to provide adequate counting statistics, with pixel size set generally by the beam size. Obtaining adequate counting statistics, especially for trace elements, is aided by the large collection solid-angle of Maia and the pulse handling and real-time processing modules, which can handle event rates beyond $20 \mathrm{M} / \mathrm{s}$. Alternatively, large solid-angle and small dwell times can be used to minimize dose per pixel and avoid radiation damage induced changes to samples. Combined with the reliability and stability of the XFM beamline, the result is a flexible microanalytical tool for large area, high definition mapping to around $10^{8}$ pixels, and for 3D imaging modalities, such as 3D fluorescence tomography and XANES chemical state imaging.

In user applications, XFM imaging using Maia is proving especially useful for imaging and locating rare minute metal particles in geological and biological samples. The large detector array, with a wide range of X-ray take-off angles, also provides sensitivity to the depth of features within a sample, which has been utilized to provide an imaging depth contrast and to determine the depth of selected features, such as rare precious metal phases. The survey and detail approach permits added accumulated statistics for more accurate phase estimation and depth determination.

In the study of the carbonaceous chondrites and rare PGE phases in mantle derived rocks, these capabilities enable these particles to be located in a large area sample, and in depth. Further analysis of fine-scale 'hot-spot' element associations in Allende, and other more primitive carbonaceous chondrites, supported by techniques involving focused ion beam analysis of extracted slices over specific hot-spots, will allow us to determine which grains are primary condensates and which are the result of secondary processes. Constraining the composition and mineralogy of primary condensates would provide a window into a variety of protoplanetary disk processes.

\section{Acknowledgements}

This research was undertaken on the X-ray fluorescence microscopy beamline at the Australian Synchrotron, Victoria, Australia.

\section{REFERENCES}

[1] Kirkham, R., Dunn, P.A., Kucziewski, A., Siddons, D.P., Dodanwela, R., Moorhead, G., Ryan, C.G., De Geronimo, G., Beuttenmuller, R., Pinelli, D., Pfeffer, M., Davey, P., Jensen, M., Paterson, D., de Jonge, M.D., Kusel, M., and McKinlay, J., "The Maia Spectroscopy Detector System: Engineering For Integrated Pulse Capture, Low-Latency Scanning And Real-Time Processing," AIP Conference Proc. 1234, 240-243 (2010).

[2] Paterson, D., de Jonge, M. D., Howard, D.L., Lewis, W., McKinlay, J., Starritt, A., Kusel, M., Ryan, C.G., Kirkham, R., Moorhead, G., Siddons, D.P., "The X-ray Fluorescence Microscopy Beamline at the Australian Synchrotron," AIP Conf. Proc. 1365, 219-222 (2011).

[3] De Geronimo, G., O’Connor, P., Beuttenmuller, R.H., Li, Z., Kuczewski, A.J., and Siddons, D.P., IEEE Transactions on Nuclear Science 50, 885-891 (2003).

[4] Ryan, C.G., Kirkham, R., Hough, R.M., Moorhead, G., Siddons, D.P., de Jonge, M.D., Paterson, D.J., De Geronimo, G., Howard, D.L., and Cleverley, J.S., "Elemental X-ray imaging using the Maia detector array: The benefits and challenges of large solid-angle," Nucl. Instr. Meth A 619, 37-43 (2010).

[5] Dragone, A., De Geronimo, G., Fried, J., Kandasamy, A., O’Connor, P., and Vernon, E., "The PDD ASIC: Highly Efficient Energy and Timing Extraction for High-Rate Applications," in IEEE Nuclear Science Symposium Conference Record 2, 914-918 (2005). 
[6] Reuter, W., Luria, A., Cardone, F., and Ziegler, J.F., "Quantitative analysis of complex targets by protoninduced X-rays,” J. App. Phys. 46, 3194-3202 (1975).

[7] Ebel, H., Svagera, R., Ebel, M.F., Shaltout, A., and Hubbell, J.H., X-Ray Spectrometry 32, 442-451 (2003).

[8] Elam, W.T., Ravel, B.D., Sieber, J.R., Radiation Physics and Chemistry 63, 121-128 (2002).

[9] Campbell, J.L., Atomic Data and Nuclear Data Tables 85, 291-315 (2003).

[10] Ryan, C.G., Cousens, D.R., Sie, S.H., Griffin, W.L., Suter, G.F. and Clayton, E., Nucl. Instr. Meth. B47, 55-71 (1990).

[11] Ryan, C.G., Etschmann, B.E., Vogt, S., Maser, J., Harland, C.L., van Achterbergh, E. and Legnini, D., "Nuclear Microprobe - Synchrotron Synergy: Towards Integrated Quantitative Real-time Elemental Imaging using PIXE and SXRF," Nucl. Instr. Meth. B231, 183-188 (2005).

[12] Ryan, C.G., Siddons, D.P., Kirkham, R., Dunn, P.A., Kuczewski, A., Moorhead, G., De Geronimo, G., Paterson, D.J., de Jonge, M.D., Hough, R.M., Lintern, M.J., Howard, D.L., Kappen, P. and Cleverley, J., "The New Maia Detector System: Methods for High Definition Trace Element Imaging of Natural Material," X-Ray Optics and Microanalysis, AIP Conference Proc. 1221, 9-17 (2010).

[13] Ryan, C.G. and Jamieson, D.N., Nucl. Instr. Meth. B77, 203-214 (1993).

[14] Ryan, C.G., "Quantitative Trace Element Imaging using PIXE and the Nuclear Microprobe," International Journal of Imaging Systems and Technology, Special issue on "Advances in Quantitative Image Analysis", 11, 219-230 (2000).

[15] Ryan, C.G., Jamieson, D.N., Churms, C.L. and Pilcher, J.V., Nucl. Instr. Meth. B104, 157-165 (1995).

[16] Ryan, C.G., Siddons, D.P., Kirkham, R., Paterson, D.J., de Jonge, M.D. et al., in prep.

[17] Howard, D.L., de Jonge, M.D., Lau, D., Hay, D., Varcoe-Cocks, M., Ryan, C.G., Kirkham, R., Moorhead, G., Paterson, D. and Thurrowgood, D., "High Definition X-ray Fluorescence Elemental Mapping of Paintings," Analytical Chemistry 84, 3278-3286 (2012).

[18]Zhang, J.Z., Bryce, N.S., Siegele, R., Carter, E.A., Paterson, D., de Jonge, M.D., Howard, D.L., Ryan, C.G., Hambley, T.W., "The use of spectroscopic imaging and mapping techniques in the characterisation and study of drug response in DLD-1 cell spheroid tumour models," Integrated Biology 4, 1072-1080 (2012).

[19] Kopittke, P.M., Menzies, N.W., de Jonge, M.D., McKenna, B.A., Donner, E., Webb, R.I., Paterson, D., Ryan, C.G., Glover, C.J. and Lombi, E., "In situ distribution and speciation of toxic $\mathrm{Cu}, \mathrm{Ni}$ and $\mathrm{Zn}$ in hydrated roots of cowpea," Plant Physiology 156, 663-673 (2011).

[20] Lombi, E., de Jonge, M.D., Donner, E., Kopittke, P.M., Howard, D.L., Kirkham, R., Ryan, C.G. and Paterson, D., "Fast X-ray fluorescence microtomography of hydrated biological samples," PLoS One (Public Library of Science) 6(6): e20626 (2011).

[21] Carey, A.-M., Lombi, E., Donner, E., de Jonge, M.D., Punshon, T., Jackson, B.P., Guerinot, M.L., Price, A.H., Meharg, A.A., "A review of recent developments in the speciation and location of arsenic and selenium in rice grain," Analytical and Bioanalytical Chemistry 402, 3275-3286 (2012).

[22] Lintern, M., Hough, R. and Ryan, C., "Experimental studies on the gold-in-calcrete anomaly at Edoldeh Tank Gold Prospect, Gawler Craton, South Australia," J. Geochem. Explor. 112, 189-205 (2012).

[23] Barnes, S., Godel, B., Locmelis, M., Fiorentini, M., Ryan, C., "Extremely Ni-rich Fe-Ni sulphide assemblages in komatiitic dunite at Betheno, Western Australia: results from synchrotron X-ray fluorescence mapping," Aust. J. Earth Sciences 58, 691-709 (2011).

[24] Etschmann, B.E., Ryan, C.G., Brugger, J., Kirkham, R., Hough, R.M., Moorhead, G.F., Siddons, D.P., De Geronimo, G., Kuczewski, A., Dunn, P.A., Paterson, D.J., de Jonge, M.D., Howard, D.L., Davey, P. and Jensen, M., "Reduced As components in highly oxidized environments: Evidence from full spectral XANES imaging using the Maia massively parallel detector," American Mineralogist 95, 884-887 (2010).

[25] Brugger, J., Pring, A., Reith, F., Ryan, C.G., Etschmann, B., Liu, W., O’Neill, B., Ngothai, Y., "Probing ore deposits formation: New insights and challenges from synchrotron and neutron studies," Radiation Physics and Chemistry 79, 151-161 (2010).

[26] Hough, R.M., Noble, R.R.P., Butt, C.R.M., Ryan, C.G., Lintern, M.J., Reddy, S.M., Paterson, D., de Jonge, M.D., Smart Science For Exploration And Mining, VOL 1 and 2, 716-718 (2010).

[27] Cleverley J.S., Hough R., Ryan C., Maas R., Blake K., Nugus M., "Gold Precipitation: The Big Picture from Micro-chemical Processes," Smart Science For Exploration And Mining, VOL 1 and 2, 213-215 (2010).

[28] Padovan, A., Munksgaard, N., Alvarez, B., McGuinness, K., Parry, D., Gibb, K., "Trace metal concentrations in the tropical sponge Spheciospongia vagabunda at a sewage outfall: synchrotron X-ray imaging reveals the micron-scale distribution of accumulated metals," Hydrobiologia 687, 275-288 (2012). 
[29] Donner, E., Ryan, C.G., Howard, D.L., Zarcinas, B., Scheckel, K.G., McGrath, S.P., de Jonge, M.D., Paterson, D., Naidu, R., Lombi, E., "A multi-technique investigation of copper and zinc distribution, speciation and potential bioavailability in biosolids," Environmental Pollution 166, 57-64 (2012).

[30] Myers D.E., Ryan C.G., Kirkham R., Paterson D., Moorhead G., Dunn P.A., Siddons D.P., de Jonge M.D., Howard D.L., De Geronimo G., Altissimo M., O'Brien T.J., Jones N.C., Stevenson A.W., Mayo S., and Wilkins S., "High-definition mapping of trace metal elements in the hippocampus in a model of closed-head traumatic brain injury," Injury 41, Supplement 1, S30-S31 (2010).

[31] Lombi E., Smith E., Hansen T.H., Paterson D., de Jonge M.D., Howard D.L., Persson D.P., Husted S., Ryan C., and Schjoerring J.K.,"Megapixel imaging of (micro)nutrients in mature barley grains," J Experimental Botany 62, 273-282 (2011).

[32] Lye, J.C., Hwang, J.E.C., Paterson, D., de Jonge, M.D., Howard, D.L., Burke, R., "Detection of Genetically Altered Copper Levels in Drosophila Tissues by Synchrotron X-Ray Fluorescence Microscopy," PLoS One 6, e26867-1 to e26867-8 (2011).

[33] McColl, G., James, S.A., Mayo, S., Howard, D.L., Ryan, C.G., Kirkham, R., Moorhead, G.F., Paterson, D., de Jonge, M.D., Bush, A.I., "Caenorhabditis elegans Maintains Highly Compartmentalized Cellular Distribution of Metals and Steep Concentration Gradients of Manganese," PLoS ONE 7, e32685-1 - e32685-4 (2012).

[34] Kopittke, P.M., de Jonge, M.D., Menzies, N.W., Wang, P., Donner, E., McKenna, B.A., Paterson, D., Howard, D.L., and Lombi, E., "Examination of the Distribution of Arsenic in Hydrated and Fresh Cowpea Roots Using Two- and Three-Dimensional Techniques," Plant Physiology 159, 1149-1158 (2012).

[35] Wang, P., Menzies, N.W., Lombi, E., McKenna, B.A., de Jonge, M.D., Donner, E., Blamey, F.P.C., Ryan, C.G., Paterson, D.J., Howard, D.L., James, S.A., Kopittke, P.M., Science of the Total Environment 463-464 131-139 (2013).

[36] Macdonald, D., Rougieux, F., Mansoulie, Y., Tan, J., Paterson, D., Howard, D.L., de Jonge, M.D. and Ryan, C.G., "Scanning x-ray fluorescence microspectroscopy of metallic impurities in solar-grade silicon," Phys. Status Solidi A 207, 1807-1810 (2010).

[37] de Jonge, M.D. and Vogt, S., "Hard X-ray fluorescence tomography —an emerging tool for structural visualization," Current Opinion in Structural Biology 20, 606-614 (2010).

[38] de Jonge, M.D., pers. comm.; manuscript in prep.

[39]Dyl, K.A., Cleverley, J.S., Bland, P.A., Ryan, C.G., and Hough, R.M., "Trace element mapping of carbonaceous chondrites Allende (CV3-Oxidized) and Vigarano (CV3-Reduced) at 2-micron resolution over centimetre-scales," submitted to Geochimica et Cosmochimica Acta.

[40] Barnes, S.J., Godel, B., Ryan, C.G., "Microprospecting for platinum group minerals by X-ray fluorescence mapping using the Maia detector," Goldschmidt Geochemistry Conference, Prague, July 2011. Mineralogical Magazine 75, 489 (2011).

[41]Fonseca, R.O.C., Laurenz, V., Mallmann, G., Luguet, A., Hoehne, N., Jochum, K.P., "New constraints on the genesis and long-term stability of Os-rich alloys in the Earth's mantle," Geochimica et Cosmochimica Acta 87, 227 (2012).

[42] Maier, W.D., Rasmussen, B., Fletcher, I., Yang, S., "Direct precipitation of Pt alloys from basaltic magma in the 2.77 Ga Monts de Cristal Complex, Gabon," $12^{\text {th }}$ International Ni-Cu-PGE Symposium, Guiyang, China, 106-109 (2012).

[43]Fonseca, R.O.C., Campbell, I.H., O'Neill, H.S.C., Allen, C.M., "Solubility of Pt in sulphide mattes: Implications for the genesis of PGE-rich horizons in layered intrusions," Geochimica et Cosmochimica Acta. 73, 5764-5777 (2009).

[44] Barnes, S.J., Godel, B., Gürer, D., Brenan, J.M., Robertson, J., Paterson, D., "Sulfide-olivine Fe-Ni exchange and the origin of anomalously Ni-rich magmatic sulfides," Econ. Geol. 108, in press (2013). 